\title{
Phenotype of autosomal recessive congenital microphthalmia mapping to chromosome $14 \mathrm{q} 32$
}

David A R Bessant, Khalid Anwar, Shagufta Khaliq, Abdul Hameed, M Ismail,
Annette M Payne, S Qasim Mehdi, Shomi S Bhattacharya
Department of

Molecular Genetics, Institute of Ophthalmology,

London

D A R Bessant

A M Payne

S S Bhattacharya

Moorfields Eye Hospital, London D A R Bessant

Dr A Q Khan Research Laboratories, Biomedical and

Genetic Engineering Division, Islamabad,

Pakistan

K Anwar

S Khaliq

A Hameed

$M$ Ismail

S Q Mehdi

Correspondence to: David A R Bessant,

Department of Molecular Genetics, Institute of Ophthalmology, London EC1V 9EL.

Accepted for publication 8 March 1999

\begin{abstract}
Background-Congenital microphthalmia (OMIM: 309700) may occur in isolation or in association with a variety of systemic malformations. Isolated microphthalmia may be inherited as an autosomal dominant, an autosomal recessive, or an $\mathrm{X}$ linked trait.

Methods-Based on a whole genome linkage analysis, in a six generation consanguineous family with autosomal recessive inheritance, the first locus for isolated microphthalmia was mapped to chromosome 14q32. Eight members of this family underwent clinical examination to determine the nature of the microphthalmia phenotype associated with this locus.

Results-All affected individuals in this family suffered from bilateral microphthalmia in association with anterior segment abnormalities, and the best visual acuity achieved was "perception of light". Corneal changes included partial or complete congenital sclerocornea, and the later development of corneal vascularisation and anterior staphyloma. Intraocular pressure, as measured by Schiotz tonometry, was greatly elevated in many cases.

Conclusions-This combination of ocular defects suggests an embryological disorder involving tissues derived from both the neuroectoderm and neural crest. Other families with defects in the microphthalmia gene located on 14q32 may have a similar ocular phenotype aiding their identification.

(Br F Ophthalmol 1999;83:919-922)
\end{abstract}

Congenital microphthalmia (microphthalmia) is a common ocular malformation in which a small, and sometimes malformed, eye is presumed to develop as a result of incomplete invagination of the optic vesicle into the optic cup, or defective closure of the optic vesicle, occurring between the second and sixth weeks of gestation. Both the extreme form of microphthalmia, anophthalmia, and more minor degrees of microphthalmia have been observed in the same family indicating that they should be considered as related conditions. ${ }^{1}$ The reported prevalence at birth of these conditions has varied between 0.22 and 5.9 per 10000 , due, at least in part, to great variability in the inclusion criteria for microphthalmia used in different registers. ${ }^{23}$ The largest study (of close to 5.7 million births) reported a prevalence of 1.5 per $10000{ }^{4}$
Up to $80 \%$ of cases of microphthalmia occur as part of syndromes that include systemic malformations, especially cardiac defects, facial clefts, microcephaly, and hydrocephaly. ${ }^{4}$ These include over 100 genetic traits (autosomal dominant, autosomal recessive, and sex linked) and deletions or translocations of virtually all the chromosomes ${ }^{1}$ (Online Mendelian Inheritance in Man, http:// www.ncbi.nlm.nih.gov/OMIM/). Not all cases of microphthalmia are genetic and both maternal virus infections (for example, rubella and cytomegalovirus) and drug use (for example, alcohol, thalidomide, and retinoic acid) have been implicated in the development of microphthalmia in some patients. ${ }^{1}$

Isolated microphthalmia is frequently associated with other ocular abnormalities including coloboma and cataract. ${ }^{1}$ It may be inherited as an autosomal dominant, ${ }^{56}$ an autosomal recessive, ${ }^{78}$ or an X linked trait. ${ }^{9}$

Sclerocornea (OMIM: 269400) is a congenital malformation of the cornea, such that the boundary between the cornea and the sclera is obscured. Usually the involvement is limited to the peripheral part of the cornea, but it may extend to the entire cornea. The mild form has been reported as a dominant trait, and the severe form as a recessive trait. ${ }^{10}$ It has been described in association with isolated microphthalmia in three pedigrees, ${ }^{511}$ and as part of the MIDAS (microphthalmia, dermal aplasia, and sclerocornea) syndrome, which may be seen in females with an Xp22 deletion. ${ }^{12}$

We have recently identified the first genetic locus for isolated microphthalmia to $14 \mathrm{q} 32$ by homozygosity mapping in a large inbred autosomal recessive pedigree. ${ }^{13}$ This paper describes the microphthalmia phenotype observed in that family.

\section{Phenotypic characterisation}

A six generation consanguineous family in which eight living members, ranging in age from 1 to 48 years, were affected by isolated microphthalmia, was identified in Pakistan (Fig 1). Ophthalmological examination of the parents of the affected individuals revealed evidence of microphthalmia in only one case, indicating an autosomal recessive mode of inheritance (the father of patient VI:4 is affected, but he is the product of a consanguineous marriage and also has three affected siblings). All family members underwent a full ophthalmic and general physical examination by a local clinician (KA). Five patients, including those in whom slit lamp biomicroscopy 


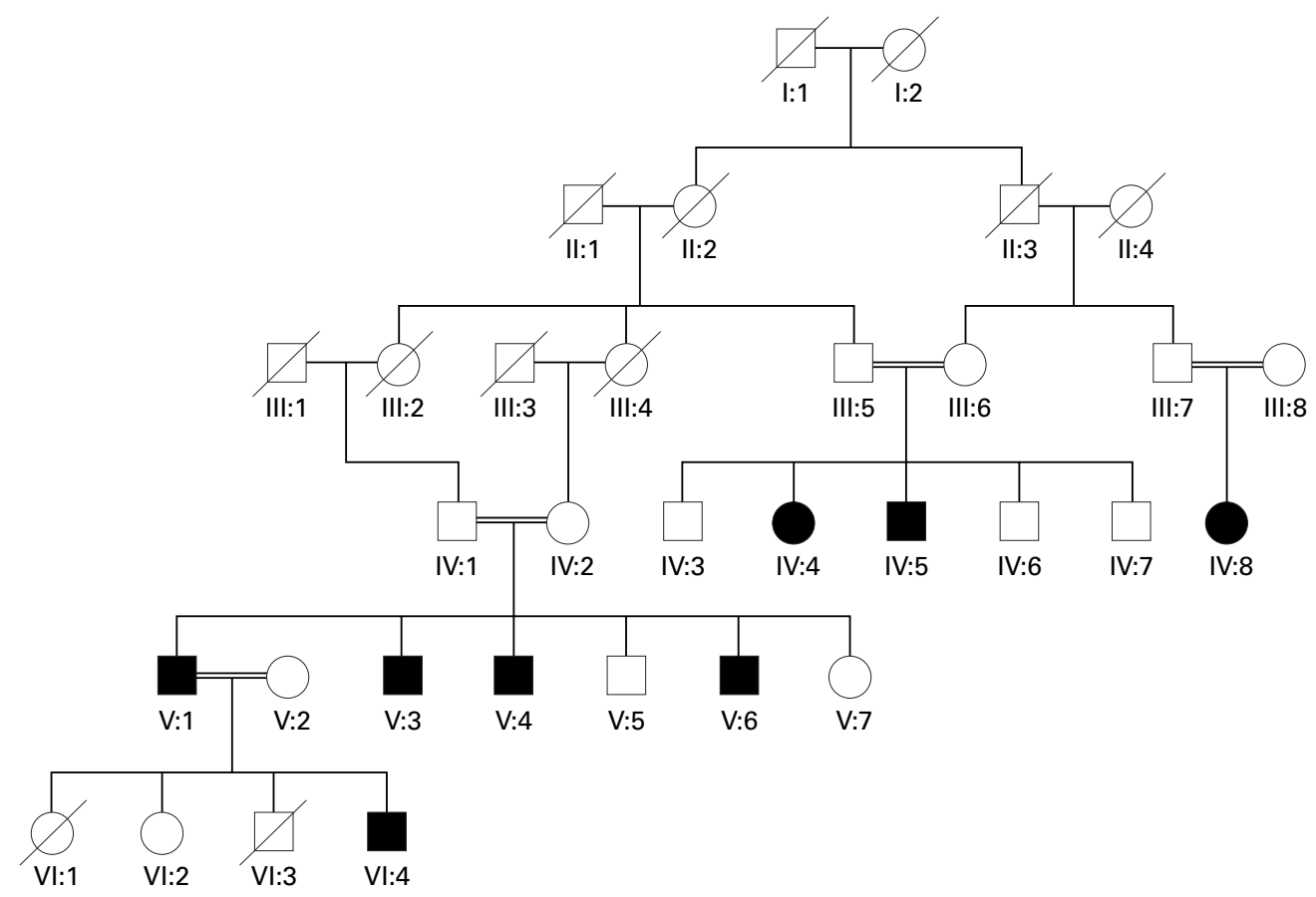

Figure 1 Pedigree of the autosomal recessive microphthalmia family, previously linked to chromosome 14q32, with affected individuals denoted by solid symbols. Individual III:8 is, in fact, the sister of IV:2. The marriage of V:1 and V:2 is consanguineous through the family of individual III:1 (V:1's paternal grandfather). Since he is not recorded as a descendant of $I: 1$ and I:2, this does not help to explain the transmission of two disease alleles to VI:4.

demonstrated complete sclerocornea, also underwent ultrasonography (Mideck Echoscan model US3300) to determine the axial length and the status of the posterior segment of the eye. No associated systemic abnormality or evidence of mental retardation could be found in any affected individual.

The ocular phenotype in affected individuals is summarised in Table 1. All patients had bilateral microphthalmia, corneal opacity, and nystagmus. Visual acuity was "perception of light" in five cases and "no perception of light" in the remaining three. Axial lengths, measured by ultrasonography, varied from 8.0 to 20.8 $\mathrm{mm}$. Those eyes with axial lengths greater than $16 \mathrm{~mm}$ were all affected by anterior staphylomata. ${ }^{3}$ The degree of corneal opacity present permitted visual assessment of the crystalline lens and the fundus in only one case.

In those eyes with an identifiable cornea, its diameter was reduced in the majority of cases $(8-12 \mathrm{~mm})$. In three brothers $(\mathrm{V}: 1, \mathrm{~V}: 3$, and $\mathrm{V}: 6)$ an anterior staphyloma was present in at least one eye making the assessment of corneal diameter equivocal. These staphylomata were

Table 1 Clinical features of affected members of microphthalmia family

\begin{tabular}{llllll}
\hline $\begin{array}{l}\text { Patient } \\
\text { No }\end{array}$ & $\begin{array}{l}\text { Age } \\
\text { (years) }\end{array}$ & $\begin{array}{l}\text { Visual } \\
\text { acuity }\end{array}$ & $\begin{array}{l}\text { Axial length } \\
(m m, R / L)\end{array}$ & Corneal involvement & IOP $(R / L)$ \\
\hline VI:4 & 1 & PL & $14.6 / 15.4$ & opaque & $41.5 / 41.5$ \\
V:6 & 28 & PL & $15.9 / 20.8$ & opaque, vascularised, anterior staphyloma & $34.5 / 41.5$ \\
V:4 & 33 & no PL & $14.7 / 8.0$ & opaque, vascularised, left sclerocornea & $41.5 / 41.5$ \\
V:3 & 35 & no PL & - & opaque, vascularised, anterior staphyloma & $41.5 / 41.5$ \\
V:1 & 39 & PL & $19.2 / 18.2$ & opaque, vascularised, anterior staphyloma & $12.2 / 17.3$ \\
IV:8 & 40 & PL & $10.0 / 8.5$ & complete sclerocornea right/left & $17.3 / 17.3$ \\
IV:5 & 41 & no PL & - & opaque, vascularised & $41.5 / 17.3$ \\
IV:4 & 48 & PL & - & opaque, vascularised & $17.3 / 10.2$
\end{tabular}

$\mathrm{PL}=$ perception of light; IOP $=$ intraocular pressure measured by Schiotz tonometry and converted to $\mathrm{mm} \mathrm{Hg}$. described as developing during early adult life and were not congenital.

In subject VI:4, a 1 year old boy, the cornea was opaque and avascular, with a clearly defined limbus (Fig 2A). This individual had less corneal opacity than other members of his family, and this permitted examination of the lens, which was clear and not subluxed, and the iris, which appeared normal. While corneal opacity precluded direct examination of the retina, B-scan ultrasonography did not indicate any structural abnormality of the posterior segment. In older patients severe opacification of the corneal was associated with varying degrees of corneal vascularisation. This vascularisation was reported by all members of the family to have occurred during childhood and not to have been present at birth (Fig 2B).

In two cases clinical examination revealed extreme microphthalmia and complete sclerocornea. In patient IV:8 ultrasonography confirmed bilateral microphthalmia, with axial lengths of $10.0 \mathrm{~mm}$ and $8.5 \mathrm{~mm}$ for the right and left eyes respectively (Table 1), and demonstrated the presence of an apparently normal iris, lens, and vitreous cavity. The right eye of patient $\mathrm{V}: 4$ had moderate corneal opacity and an axial length of $14.7 \mathrm{~mm}$ (Fig 2C), while his completely opaque left eye had an axial length of $8.0 \mathrm{~mm}$ (Fig 2D). In this pedigree, therefore, the smallest eyes were also those with the greatest degree of corneal opacity. Patient V:1, who had bilateral anterior staphylomata (and axial lengths of 18.2 and $19.2 \mathrm{~mm}$ ), also underwent ultrasonography, and this again confirmed that the lens, iris, and posterior segment were grossly intact. 


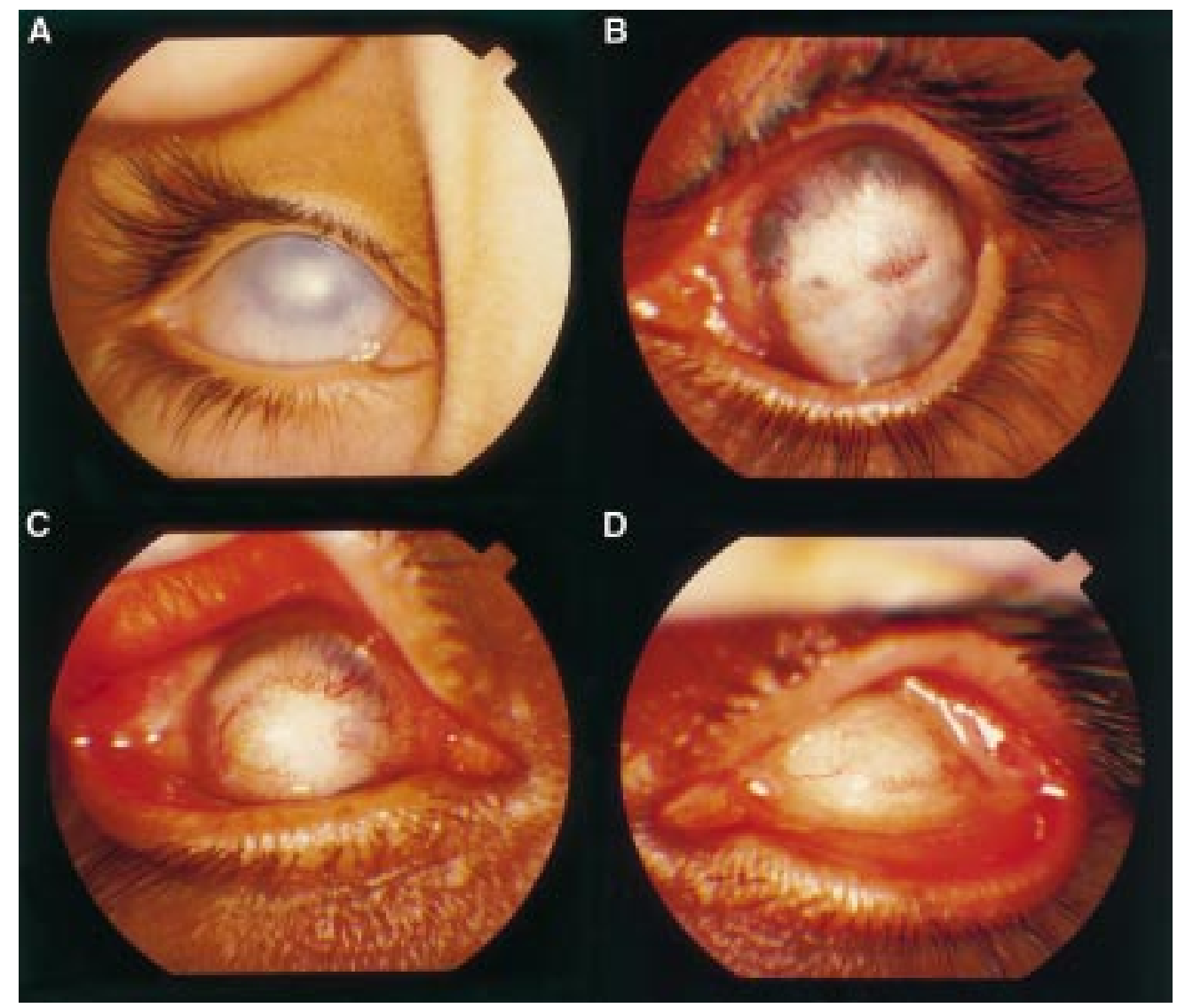

Figure 2 (A) Patient VI:4 (aged 1 year), right eye demonstrating microphthalmia with corneal opacity, but no vascularisation and a clearly defined limbus. (B) Patient V:6 (aged 28 years), left eye with corneal vascularisation and an anterior staphyloma. (C) and (D) Right and left eyes of patient V:4 showing left severe microphthalmia and total sclerocornea. Intraocular pressure, as assessed by standardised Schiotz tonometry, was markedly elevated in five patients (nine out of 16 eyes), including the 1 year old boy (VI:4).

\section{Discussion}

The phenotype seen in this $14 \mathrm{q} 32$ linked family comprises varying degrees of microphthalmia in association with severe anterior segment abnormalities. There is, however, no evidence of coloboma or of anomalies of the lens, vitreous, or retina. While it is possible that altered scleral rigidity in these abnormal eyes may have rendered the Schiotz tonometer readings somewhat inaccurate it seems certain that raised intraocular pressure forms part of this microphthalmia phenotype. Since highly elevated intraocular pressures may be observed even at the age of 1 year it is possible that this may contribute significantly to the progressive corneal changes observed including staphyloma formation. The marked corneal opacity observed at this age (Fig 2A) may be due in part to the raised intraocular pressure, but appears rather too severe to be caused by this alone. Since three eyes belonging to older family members were found to have total sclerocornea it seems likely that corneal changes are a primary part of the microphthalmia phenotype and not solely the result of raised intraocular pressure.

The association of microphthalmia with coloboma and cataract is well recognised. Corneal opacity has, however, only been recorded in three isolated microphthalmia families, one of which demonstrated autosomal dominant inheritance, one recessive, and one $\mathrm{X}$ linked. ${ }^{5911}$ One of these, a family from India consisting of four affected children of normal parents, was reported to have a similar phenotype to that seen in our pedigree. ${ }^{11}$ Three of these siblings, aged between 8 and 14 years, were examined. All had bilateral microphthalmia, nystagmus, and anterior segment abnormalities. The eldest had an extremely microphthalmic left eye, with no evidence of a posterior segment on A-scan ultrasonography, and partial sclerocornea in the right eye. The second had no corneal anomalies, but did have bilateral congenital cataracts. The youngest had bilateral total sclerocornea, corneal vascularisation, and left congenital anterior staphyloma.

The range of anterior segment changes seen in this family is similar to that observed in the 14q32 linked family, and the mode of inheritance is identical. This apparent similarity might suggest a common genetic basis for the disorder in both families. It must be noted, however, that none of our family had microphthalmia and cataract in the absence of corneal changes, and that both corneal vascularisation and staphyloma formation were progressive degenerative processes in the $14 \mathrm{q} 32$ linked family, in complete contrast with the family reported by Ghose in whom they were congenital. ${ }^{11}$

Congenital microphthalmia associated with anterior segment anomalies and raised in- 
traocular pressure represents a phenotype which may have important implications for the molecular biology of human eye development. This combination indicates an embryological defect involving both the optic cup, derived from neuroectoderm, and neural crest tissue from which the corneal stroma and endothelium, anterior iris stroma, ciliary body, sclera, and portions of trabecular meshwork, including endothelium, develop. ${ }^{14}$ Abnormal migration and/or proliferation of neural crest cells has been implicated in the development of several anterior segment dysgenesis and congenital glaucoma syndromes. ${ }^{1}$

At least 14 transcription factors that are essential for normal mammalian eye development have been described, along with 32 other tissue restricted transcription factors that are expressed at some stage in the developing or mature eye. ${ }^{16}{ }^{17}$ Some of the human genes are homologues of Drosophila eye development genes (for example, eyeless, PAX6; sine oculis, Six3; and eyes absent, Eya), or of genes which have been shown to give rise to microphthalmia in mouse models (ocular retardation, CHX10; and microphthalmia, MITF). Four transcription factors ( $P A X 2, P A X 3, P A X 6$, and $C R X$ ) have been associated with ocular disease in humans, but none has been associated with isolated human microphthalmia. ${ }^{18-21}$

The $14 \mathrm{q} 32$ region, at the telomere of human chromosome $14 \mathrm{q}$, is syntenic with a region of mouse chromosome 12 which contains a number of transcription factors, some of which are important in eye development. However, the human homologues of these genes can be excluded as candidates for $14 \mathrm{q} 32$ linked microphthalmia since they map outside the critical disease region defined by the observed recombination events. ${ }^{13}$ This suggests that this disease is due to mutations in a novel developmental gene that may be critical for eye development.

We would like to thank the family members for taking part in this study. This collaborative effort was made possible by Well- come Trust Grant No 049571/Z/96/Z. Drs David A R Bessant and Annette M Payne are supported by the Medical Research Council of the UK (grant No G9301094).

1 Warburg M. Classification of microphthalmos and coloboma. F Med Genet 1993;30:664-9.

2 Castilla E. Clusters of anophthalmia. No further clues from global investigations. BMF 1994;305:206.

3 Stoll C, Alembik Y, Dott B, et al. Epidemiology of congenital eye malformations in 131,760 consecutive births. Ophthalmic Paediatr Genet 1992;13:179-86.

4 Kallen B, Robert E, Harris J. The descriptive epidemiology of anophthalmia and microphthalmia. Int $\mathcal{f}$ Epidemiol 1996;25:1009-16.

5 Pearce WG. Corneal involvement in autosomal dominant coloboma/microphthalmos. Can $\mathcal{f}$ Ophthalmol 1986;21: 291-4.

6 Vingolo EM, Steindl K, Forte R, et al. Autosomal dominant simple microphthalmos. I Med Genet 1994;31:721-5.

7 Kohn G, El Shawwa R, El Rayyes E. Isolated 'clinical anophthalmia' in an extensively affected Arab kindred. Clin Genet 1988;33:321-4.

8 Zlotogora J, Legum C, Raz J, et al. Autosomal recessive colobomatous microphthalmia. Am f Med Genet 1994;49: 261-2.

9 Stephens FE. A case of sex-linked microphthalmia. 7 Hered 1947;38:307-10.

10 Elliott JH, Feman SS, O'Day DM, et al. Hereditary sclerocornea. Arch Ophthalmol 1985;103:676-9.

11 Ghose S, Singh NP, Kaur D, et al. Microphthalmos and anterior segment dysgenesis in a family. Ophthamic Paediat Genet 1991;12:177-82.

12 Happle R, Daniels O, Koopman RJ. MIDAS syndrome (microphthalmia, dermal aplasia, and sclerocornea): an X-linked phenotype distinct from Goltz syndrome. Am $\mathcal{F}$ X-linked phenotype distinct

13 Bessant DAR, Khaliq S, Hameed A, et al. A locus for autosomal recessive congenital microphthalmia maps to chromosome 14q32. Am F Hum Genet 1998;62: 1113-16.

14 Beauchamp GR, Knepper PA. Role of the neural crest in anterior segment development and disease. 7 Pediatr Ophthalmol Strabismus 1984;21:209-14.

15 Bahn CF, Falls HF, Varley GA, et al. Classification of corneal endothelial disorders based on neural crest origin. Ophthalmology 1984;91:558-63.

16 Freund C, Horsford DJ, McInnes RR. Transcription factor genes and the developing eye: a genetic perspective. Hum Mol Genet 1996;5:1471-88.

17 Graw J. Genetic aspects of eye development in vertebrates. Dev Genet 1996;18:181-97.

18 Sanyanusin P, Schimmenti L, McNoe LA, et al. Mutation of the PAX2 gene in a family with optic nerve colobomas, renalanomalies and vesicoureteral reflux. Nature Genet 1995;9: 358-64.

19 Tassabehii M, Read AP, Newton VE, et al. Waardenburg's syndrome patients have mutations in the human homosyndrome patients have mutations in the human homo-
logue of the Pax-3 paired box gene. Nature 1992;355:6356.

20 Hanson I, Van Heyningen V. Pax6: more than meets the eye. Trends Genet 1995;11:268-72.

21 Freund CL, Gregory Evans CY, Furukawa T, et al. Cone-rod dystrophy due to mutations in a novel photoreceptor-specific homeobox gene (CRX) essential for maintenance of the photoreceptor. Cell 1997;91:543-53. 